\title{
Los derechos propios de los pueblos étnicos en el Acuerdo de Paz de agosto de $2016^{* *}$
}

\section{The own rights of ethnical people in the Colombian Peace Agreement of August 2016}

RESUMEN

Los Acuerdos de Paz firmados en La Habana entre el Gobierno colombiano y las Fuerzas Armadas Revolucionarias de Colombia - Ejército del Pueblo (FARC-EP), poniendo fin a un conflicto de más de cincuenta años, prevén mecanismos de justicia transicional adaptados a víctimas provenientes de grupos étnicos y culturales. En efecto, las modalidades de reparación de estas víctimas toman en cuenta sus particularidades étnicas, culturales y sociales, así como sus vivencias del conflicto y sus necesidades específicas. A pesar de que los representantes indígenas, afrocolombianos y roms hayan sido invitados tardíamente a las conversaciones de paz en La Habana, y de que su poca visibilidad en el proceso haya dejado presagiar una neutralización de su victimización peculiar, el "enfoque diferencial" y el "Capítulo étnico" incluidos en el Acuerdo Final anuncian algunos logros para estas comunidades en Colombia: primero, su reconocimiento como principales víctimas del conflicto; luego, la afirmación de la idea según la cual grupos étnico disponen de derechos específicos; y sobre todo, la posibilidad para ellos de apropiarse el derecho para adaptarlo a sus cosmovisiones.

Preparación de Doctorado en l'École des Hautes Études en Sciences Sociales (EHEss, Francia), maestría en Derechos Humanos de la Universidad Paris Nanterre-La Défense, Francia, Abogada de la Universidad Pontificia Bolivariana de Medellín, Colombia. Contacto: 1braconnier. moreno@gmail.com

Este artículo es el resultado de una investigacion realizada entre marzo y diciembre de 2016 en el marco de la Maestría en Derechos Humanos de Paris X-Nanterre. Agradezco a la profesora Véronique Champeil-Desplats por su supervisión a lo largo de este proceso.

Recibido el 24 de junio de 2017, aprobado el 15 de octubre de 2017.

Para citar el artículo: Braconnier Moreno, L. Los derechos propios de los pueblos étnicos en el Acuerdo de Paz de agosto de 2016. Derecho del Estado n. ${ }^{\circ} 40$, Universidad Externado de Colombia, enero-junio de 2018, pp. 113-126. DOI: https://doi.org/10.18601/01229893.n40.05 
PALABRAS CLAVE

Acuerdo de Paz en Colombia, pluralismo jurídico, jurisdicción indígena, justicia transicional, Capítulo étnico, jurisdicciones especiales para la paz.

\section{ABSTRACT}

The Peace Agreement signed up in Havana between the Colombian government and the Fuerzas Armadas Revolucionarias de Colombia - Ejército del Pueblo (FARC-EP), ending a conflict of more than fifty years, establishes transitional justice mechanisms adapted to victims from ethnic and cultural groups. Indeed, modalities to repair those victims take their ethnic, cultural, social particularities into account, such as their own experiences of the conflict and special needs. Despite the fact that Indigenous, Afro-Colombian and Roms leaders have been invited belatedly to the peace conversations in Havana, and that their low visibility in the process suggested that their special victimisation would be ignored, the "differential approach" and the "Ethnic chapter", included in the Final Agreement, foreshadows some success for those communities in Colombia: first, their reconnaissance as the principal victims of the conflict; then, the idea that ethnic groups have their own rights; and above all, the possibility for them to appropriate those rights which may be adapted to their cosmovisions.

KEYWORDS

Colombian Peace Agreement, legal pluralism, indigenous law, transitional justice, Ethnic chapter, special jurisdiccions for peace.

SUMARIO

Introducción. 1. Espacios de diálogo y ajustes entre la justicia institucionalizada y los universos jurídicos de las "minorías" étnicas. 1.1. Las voces de las víctimas originarias de grupos minoritarios en las negociaciones de paz. 1.2. El enfoque diferencial en las "jurisdicciones especiales para la paz". 2. El enfoque diferencial en las reparaciones de las víctimas pertenecientes a minorías y en las garantías de no repetición. 2.1. Reparaciones étnicas colectivas inspiradas en los universos jurídicos de las víctimas. 2.2. La consulta previa como vector de etnorreparaciones y de garantías de no repetición. Conclusiones. Referencias. 
INTRODUCCIÓN

El Gobierno Nacional y las FARC-EP reconocen que los pueblos
étnicos [...] han sido afectados gravemente por el conflicto
armado interno y se deben propiciar las máximas garantías para el
ejercicio pleno de sus derechos humanos y colectivos en el marco
de sus propias aspiraciones, intereses y cosmovisiones.
Acuerdo Final para la terminación del conflicto y la construcción
de una paz estable y duradera del 24 de noviembre de 2016, Acuerdo Final, Capítulo étnico.

El "Acuerdo Final para la terminación del conflicto y la construcción de una paz estable y duradera" (en adelante "Acuerdo Final" o "Acuerdo"), firmado en el segundo semestre de 2016 entre las Fuerzas Armadas Revolucionarias de Colombia - Ejército del Pueblo (FARC-EP) y el Gobierno colombiano, prevé el fin de uno de los conflictos más antiguo del mundo, el cual produjo siete millones de desplazados internos ${ }^{1}$. Aunque los pueblos indígenas y afrodescendientes representan solamente el $13 \%$ de la población colombiana, más de la mitad de las víctimas pertenecen a estos grupos ${ }^{2}$. Mientras los debates se han enfocado en el porvenir de los guerrilleros que entregarían las armas -especialmente en los compromisos alcanzados entre amnistías y penas de prisión (Gutiérrez Ramirez, 2015)--, cabe subrayar el espacio conferido a las víctimas en esta transición, y a fortiori a las víctimas pertenecientes a grupos étnicos y culturales. Su representación desproporcionada entre las víctimas del conflicto ${ }^{3}$ se ha añadido a su invisibilización históricam (Gutiérrez Quevedo, 2012). Asimismo, su reconocimiento como víctimas implica que sus voces sean tomadas en cuenta en la construcción de la sociedad del posconflicto.

El "Paro agrario, étnico y popular" adelantado por sectores rurales en el primer semestre de 2016, durante los últimos meses de las negociaciones de paz, buscó impactar en los acuerdos con sus voces y discursos plurales. Más allá de pedir el fin de la ocupación de sus territorios y reparaciones adecuadas, las comunidades reivindicaron su derecho a la autodeterminación y la inclusión de su cosmovisión ${ }^{4}$ en el discurso sobre la paz. El desafío de imprimir en el texto final sus normatividades específicas era considerable, en

1 Estas negociaciones de paz tuvieron lugar en La Habana, Cuba, entre octubre de 2012 y finales de 2016.

2 A modo de ejemplo, el $67 \%$ de las víctimas del desplazamiento forzado en 2012 y el 44\% en 2013 pertenecen a comunidades afrodescendientes e indígenas; 35 grupos indígenas entre los 102 registrados en el territorio colombiano se encuentran en peligro de extinción a causa del conflicto armado y del desplazamiento forzado según el ACNuR y la Corte Constitucional colombiana en sus autos 004 de 2009 y 382 de 2010.

3 Las FARC, pero también los paramilitares, las Bacrim y el Ejército Nacional son responsables de masacres. El Estado colombiano fue condenado por la Corte Interamericana de Derechos Humanos (véase Masacre de Mapiripán vs. Colombia, 2005; Masacre de La Rochela vs. Colombia, 2007, etc.).

4 Concepción de integración hombre/medio ambiente a partir de la cual los seres humanos 
la medida en que los contrastes entre los universos axiológicos y normativos de los grupos minoritarios y de las instituciones estatales tienden a falsear sus interacciones.

Además de las jurisdicciones especiales indígenas, reconocidas constitucionalmente desde 1991, varias fuentes de normas informales componen el paisaje jurídico colombiano (García Villegas y de Sousa Santos 2001). Las comunidades afrodescendientes, negras, palenqueras y raizales ${ }^{5}$ obedecen más a sus propias costumbres y reglas tradicionales que a las leyes nacionales para resolver buena parte de sus litigios. Por su lado, los grupos armados ilegales -guerrillas, paramilitares y bandas criminales- siguen imponiendo sus normas en importantes zonas rurales y periurbanas del país. Así es como las interacciones entre miembros de una misma comunidad suelen someterse más a reglas informales que al derecho positivo colombiano. El simbolismo inherente a las normatividades de ciertas minorías, que alude a lo mágico, lo espiritual o lo sagrado, hizo que sus discursos fueran poco inteligibles para los negociadores. De la misma forma, para estos grupos, los documentos institucionales sobre los procesos de reparación de las víctimas han podido ser instrumentos oscuros e inaccesibles. Además, las negociaciones fueron llevadas a cabo en una tradición de ignorancia recíproca y de minimización por parte del Estado colombiano de las normatividades paralelas; desde las primeras "repúblicas independientes", las élites no consideran las prácticas de regulación social de los autóctonos y de los descendientes de esclavos como reglas legitimas (Valencia Villa, 2001).

Sin embargo, estas normas comunitarias tienden a volverse "solubles" (Ocqueteau y Belley, 1997) en el derecho específico de la transición hacia la paz. La justicia transicional, en la búsqueda de protagonismo para las víctimas, de diálogo, de reconciliación entre víctimas y victimarios, y de reparación integral, puede hacer uso de las justicias comunitarias y de las normas ad hoc de cada territorio (Lefranc, 2013). También es susceptible de inspirarse en la justicia restaurativa cuyo objetivo principal es la construcción de soluciones consensuales, basadas en el diálogo, para restablecer sus derechos o avanzar hacia una nueva configuración más inclusiva e igualitaria. Los instrumentos de justicia transicional, como estándares que se adaptan a los diferentes contextos y que pueden tejer lazos entre normatividades estatales e informales, revisten diferentes aspectos: dispositivos jurisdiccionales excepcionales, espacios de expresión de las diferentes verdades, reparación integral de las víctimas y mecanismos de no repetición. El análisis de cada uno de estos instrumentos según un enfoque diferencial demuestra en qué

y los elementos naturales son considerados como elementos interdependientes que conforman un todo.

5 Grupos étnicos y culturales reconocidos por la Constitución colombiana de 1991. 
medida pueden inspirarse en las normatividades paralelas, o bien permitir a las comunidades una apropiación del derecho mayoritario.

Además de este enfoque diferencial, el Capítulo étnico, incorporado in extremis el día de la firma del Acuerdo Final, supone un reconocimiento de derechos específicos para las minorías étnicas y culturales en esta transición. Este capítulo busca propiciar en cuatro páginas -sobre las veinte que había elaborado la Comisión étnica - garantías especiales en cada uno de los puntos acordados sobre el desarrollo agrario, la participación política, los cultivos ilícitos, la seguridad, el cese al fuego y la reparación de las víctimas. Por medio del enfoque diferencial y del análisis de este Capítulo étnico buscamos identificar en qué medida la justicia transicional que se está construyendo desde la institucionalidad colombiana integra los derechos propios de las comunidades étnicas y culturales.

La incursión del pluralismo jurídico en los Acuerdos de Paz se manifiesta en la inclusión de espacios de diálogo entre la justicia institucionalizada y los universos jurídicos de las minorías étnicas y culturales (1). Este alcance de las normatividades paralelas conlleva reparaciones y medidas de no repetición adaptadas a las reivindicaciones de los grupos étnicos (2).

\section{ESPACIOS DE DIÁLOGO Y AJUSTES ENTRE LA JUSTICIA \\ INSTITUCIONALIZADA Y LOS UNIVERSOS JURÍDICOS \\ DE LAS “MINORÍAS” ÉTNICAS}

Las negociaciones han dado cabida a una lógica pluralista, la cual supone el reconocimiento de diferentes verdades según los sectores de la población (A) y desemboca en el reconocimiento de un enfoque diferencial dentro de las jurisdicciones especiales (B).

\subsection{Las voces de las víctimas originarias de grupos minoritarios en las negociaciones de paz}

Con el propósito de poner a las víctimas en el centro del proceso de paz, los negociadores convocaron seis delegaciones de víctimas a la mesa de conversaciones de La Habana. Esta participación aportó voces que se sumaron a las voces de las partes del conflicto, afirmando reivindicaciones con sus propios lenguajes, diferentes de los lenguajes de las FARC y de los representantes del Gobierno. A pesar de sus repetidas solicitudes, las comunidades étnicas y culturales tuvieron que esperar cuatro años antes de ser invitadas, y solo pudieron participar en las discusiones acerca del sexto y último punto de las negociaciones, sobre las modalidades de ratificación de los Acuerdos.

Parte de la población civil percibió esta invitación tardía como una instrumentalización de los grupos minoritarios para avalar la legitimidad del proceso. Según el sociólogo y líder comunitario antioqueño Óscar Cárdenas, 
"[i]ndependientemente de que hayan ido o no víctimas, yo sí creo que allí faltó,

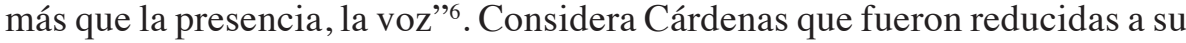
carácter de víctimas de las guerrillas, del paramilitarismo o de los crímenes de Estado -rol que les asignaron sus propios victimarios, legitimados como partes de la negociación. De esta forma, las víctimas fueron despojadas de la posibilidad de participar activamente en las decisiones, y su impacto parece haber sido de menor incidencia que el de las minorías sexuales. Asimismo, una subcomisión de género tuvo la facultad de repasar cada capítulo del Acuerdo para asegurar que el enfoque diferencial de género fuera respetado en cada punto.

Después de su visita a La Habana, los representantes de los pueblos indígenas y grupos afrodescendientes constituyeron una comisión étnica y redactaron un "Capítulo étnico" destinado a ser integrado al Acuerdo. Su propósito fue incluir "acciones y medidas para generar garantías de no repetición y la construcción de una paz desde la diversidad, los derechos colectivos y las reparaciones integrales y diferenciales" (ONIC, 2016). Sin embargo, la propuesta de estos sectores fue ignorada hasta el último momento por la mesa de conversación. El día de la firma del Acuerdo Final entre las FARC-EP y el Gobierno, al no recibir respuesta por parte de los negociadores, las organizaciones indígenas declararon que se constituían en asamblea permanente y enviaron de nuevo representantes a La Habana para ejercer una presión pacífica alrededor de la mesa. Consideraron que las partes del conflicto estaban perdiendo una "oportunidad histórica para cerrar el capítulo de un pasado de opresión”, al ignorar su propuesta (ONIC, 2016). Según lo concedió un representante de las FARC, fue solo gracias a la perseverancia de las organizaciones que el Capítulo étnico fue integrado a última hora en el Acuerdo Final ${ }^{7}$.

Varias medidas significativas fueron integradas de esta manera para subsanar carencias sistémicas en cuanto a la participación política de las minorías, y más allá, de todo tipo de oposición. El segundo punto de los Acuerdos, en cuanto a la apertura democrática y pluralista, previó asimismo las circunscripciones especiales de paz. Este mecanismo garantiza a los movimientos armados de oposición, una vez reconstituidos en movimientos legales, el derecho a ventajas políticas y a garantías para participar en las elecciones locales (Acuerdo Final del 24 de agosto de 2016, punto 6.3.12.1, p. 181). Esta medida, crucial para convencer a los guerrilleros de dejar las armas, no remite únicamente a ellos; el Capítulo étnico prevé la inclusión de candidatos pertenecientes a pueblos autóctonos en la lista de las circunscripciones

6 Entrevista con Óscar Manuel Cárdenas Avendaño, sociólogo de la Universidad de Antioquia y líder comunitario, miembro de "Raíces", colectivo de memoria histórica del barrio La Cruz de Manrique, Medellín, Colombia, 6 de julio de 2016.

7 http://www.verdadabierta.com/procesos-de-paz/farc/6377-indigenas-y-afros-a-laexpectativa, 25 de agosto de 2016. [Consultado: 26 de agosto de 2016]. 
territoriales de paz. Estas circunscripciones tendrán entonces la facultad de imponerse como tribunas políticas para las comunidades alejadas de la vida política nacional.

\subsection{El enfoque diferencial en las "jurisdicciones especiales para la paz"}

Las comisiones de verdad y las jurisdicciones especiales para la paz (en adelante JEP) constituyen dos otros instrumentos de la justicia transicional que se podrían impregnar de normatividades paralelas. El preacuerdo entre las FARC-EP y el Gobierno relativo a las víctimas solo contenía una tímida inclusión de los derechos propios de las minorías, aludiendo a un enfoque diferencial definido de manera muy evasiva. El texto sobre la Comisión de la Verdad, por ejemplo, se limita en prever: "En el desarrollo de su mandato y de sus funciones, la Comisión tendrá en cuenta las distintas experiencias, el impacto diferenciado y las condiciones peculiares de las personas en razón del sexo, género, edad, etnia, o situación de discapacidad, y de las poblaciones o sectores en condiciones de vulnerabilidad o especialmente afectados por el conflicto, entre otras" (Mesas de Conversaciones, 2016).

Además de la referencia más específica a un "enfoque étnico", el Capítulo étnico incluye garantías para las minorías. Un paso mayor hacia las jurisdicciones indígenas fue dado al concretizar la organización de la JEP. Así, como tribunales ad hoc dedicados al juicio de los antiguos guerrilleros, la JEP deberá tender hacia la búsqueda de la verdad en cuanto a los hechos vinculados con los perjuicios, y facilitar la individualización y la reparación integral de las víctimas. En el afán de fomentar la desmovilización de las FARC fueron concebidas disminuciones de penas para los guerrilleros que acepten dejar las armas y participar en el esclarecimiento de los hechos. Este mecanismo propio de la justicia transicional conlleva ciertas interrogaciones desde una perspectiva pluralista, sabiendo que ciertos frentes de la guerrilla estaban conformados mayoritariamente por miembros de comunidades indígenas o afrodescendientes. Los líderes comunitarios han estimado que en virtud del artículo 246 de la Constitución, el cual reconoce las jurisdicciones indígenas, solo estas jurisdicciones especiales son competentes para juzgar los guerrilleros de su comunidad. Han considerado en efecto que los que ya estaban condenados por estas jurisdicciones no podían nuevamente ser juzgados, esto según el principio de la autoridad de la cosa juzgada. Haciendo eco a estas reivindicaciones, el texto sobre la JEP integró una disposición según la cual "se crearán mecanismos para la articulación y coordinación con la Jurisdicción Especial Indígena según el mandato del artículo 246 de la Constitución y cuando corresponda, con la autoridades ancestrales afrocolombianas" (Acuerdo Final, punto 6.2.3.e, p. 207). Por otra parte, el Acuerdo prevé que los ex guerrilleros pertenecientes a pueblos indígenas podrán elegir volver a 
sus comunidades en vez de acudir a las zonas previstas para los combatientes desmovilizados.

Para materializar estas disponiciones, el artículo transitorio transitorio 9 incorporado a la Constitución de 1991 en virtud del Acto Legislativo 01 del 4 de abril de 2017 prevé: "Los conflictos de competencia entre la JEP y la Jurisdicción Especial Indígena serán dirimidos por una Sala Incidental conformada por dos magistrados de las salas o secciones de la JEP no afectadas por dicho conflicto jurisdiccional y dos autoridades tradicionales del pueblo indígena que ejerció o está ejerciendo jurisdicción en el caso concreto". La idea de una repartición de competencias entre las jurisdicciones sigue siendo por lo tanto imprecisa, sobre todo al recordar que todavía existe un vacío jurídico en lo que hace a la articulación de las jurisdicciones indígenas y el sistema judicial nacional ${ }^{8}$.

Por otra parte, la cuestión de las sanciones previstas para los antiguos combatientes indígenas está eludida. No se indica si autoridades de las comunidades podrán hacer parte de los juzgados, ni si las normatividades indígenas podrán ser invocadas en la definición de las penas de los guerrilleros indígenas. Feliciano Valencia, líder indígena Nasa, subraya por ejemplo: "Se va a poner en vigencia un Tribunal de Justicia Transicional, pero no han invitado a nuestros jueces. Hay una jurisdicción especial de paz que va a trabajar de manera paralela a la jurisdicción especial indígena, pero no hay un puente que transitoriamente nos ponga a conversar" (Valencia, 2016). Siguiendo su lógica, una solución posible se podría encontrar en la doble particularidad de los guerrilleros originarios de comunidades autóctonas: por una parte, la pertenencia a una minoría étnica con propia jurisdicción constitucionalmente protegida, y por otra parte, la pertenencia a un grupo de guerrilleros sometidos a jurisdicciones especiales para el fin del conflicto. Puede entonces que la propuesta de doble derogación al sistema judicial nacional suponga la instauración en las JEP de salas reservadas a los guerrilleros indígenas, involucrando jueces de las jurisdicciones indígenas.

En fin, el punto del Capítulo étnico sobre el "Sistema Integral de Verdad, Justicia, Reparación y No Repetición" agrega que los diferentes mecanismos judiciales y extrajudiciales deberán incluir la "perspectiva étnica y cultural". Así, en la eventualidad de que los jueces indígenas no sean consultados, estas audiencias deberán incluir peritajes culturales (Ariza, 2016) en virtud de un progresivo reconocimiento de las normas propias de las minorías. Este me-

8 La Corte Constitucional colombiana, en una jurisprudencia constructiva y protectora de las jurisdicciones paralelas, rellenó un vacío jurídico imponiendo como principal límite la protección de las libertades fundamentales previstas en la Constitución y los textos internacionales sobre derechos humanos. Para una visión general de la articulación entre las jurisdicciones, véase el informe del Instituto Interamericano de Derechos Humanos, Ministerio de Justicia y el Derecho, Escuela de Derecho propio del pueblo de los Pastos. Jurisdicción Especial Indígena y diálogo entre jurisdicciones. Bogotá, 2015. 
canismo prevé la presencia de peritos culturales, sociólogos, antropólogos o historiadores del derecho capaces de traducir culturalmente los hechos y las demandas de reparación en los tribuales. Se trataría de acatar los perjuicios tal como se perciben en los universos jurídicos de las comunidades, a partir del reconocimiento de que un mismo hecho pueda tener consecuencias distintas según el contexto y la identidad de las víctimas.

\section{EL ENFOQUE DIFERENCIAL EN LAS REPARACIONES DE LAS VÍCTIMAS PERTENECIENTES A MINORÍAS Y EN LAS GARANTÍAS DE NO REPETICIÓN}

Estas inflexiones con respecto al derecho ordinario permiten invocar normas consuetudinarias y mecanismos comunitarios de terminación de conflictos anclados localmente, y así promover reparaciones integrales inspiradas de los universos jurídicos de las víctimas (A). La consulta previa de los pueblos indígenas, por su parte, constituye un vector fundamental para generar normas adaptadas y condiciones de no repetición (B).

\subsection{Reparaciones étnicas colectivas \\ inspiradas en los universos jurídicos de las víctimas}

Por sus especificidades socio-culturales, las comunidades étnicas y culturales sufrieron los daños de manera peculiar y reivindican en consecuencia reparaciones apropiadas. Por ejemplo, en virtud del valor espiritual de su tierra y territorio, el desplazamiento forzoso fue vivido como un "desarraigo", o un "arrancamiento" a la Madre Tierra, que perjudicó la supervivencia de sus culturas y tradiciones. Estos daños poseen componentes psicológicos, espirituales y morales que se añaden al componente material. Cabe enfatizar que, dado el carácter colectivo de la propiedad de las tierras indígenas, estos perjuicios fueron sufridos de manera colectiva. La "comunidad" es así un concepto central reivindicado por los mismos grupos étnicos, y remite a grupos organizados de tamaño variable que comparten un territorio, una cultura e intereses comunes. Juegan un papel intermediario entre la familia y entidades más amplias como etnias indígenas u organizaciones de la sociedad civil.

Por otra parte, los pueblos indígenas, las comunidades afrodescendientes, negras, raizales y palenqueras, así como las poblaciones campesinas, comparten la idea de una simbiosis entre la comunidad y el territorio. Algunos grupos étnicos defendieron así la idea según la cual el territorio también fue víctima directa del conflicto armado (CONAFro, 2014). Por ejemplo, los múltiples daños territoriales causados por los enfrentamientos entre grupos armados, el acaparamiento de tierras para cultivos ilícitos de coca y las fumigaciones realizadas por el Estado para destruirlos tuvieron consecuencias graves tanto para el ecosistema como para las comunidades. 
Las medidas de reparación previstas por los la Ley de Víctimas y los decretos leyes de 2011, considerados como los primeros textos de justicia transicional de este proceso de paz, tomaron en cuenta las percepciones específicas de los daños (decretos leyes 4633, 4634 y 4635 de 2011). Estos textos ratificaron en efecto el concepto de "etnorreparaciones", según el cual: "Una vez se ha establecido que una violación ha sido cometida y, por tanto, se requiere reparación, el significado de la violación y las medidas de reparación correspondientes deben ser determinadas usando el lente del grupo étnico" (Rodríguez Garavito y Lam, 2011). Así, el programa de reparación desarrollado entonces reconoce que "los pueblos indígenas por sus características étnico-territoriales, culturales y identitarias, han sufrido daños de carácter colectivo y requieren de medidas específicas para una reparación pertinente" (Decreto Ley 4633 de 2011). Las comunidades son reconocidas como "sujetos colectivos de derecho", lo que implica una "mirada holística de los daños y afectaciones que estas violaciones ocasionen" (art. 42 ibíd.).

El daño a la integridad cultural es también tomado en cuenta, y se caracteriza por "la pérdida o el deterioro de la capacidad para la reproducción cultural y la conservación y transmisión intergeneracional de su identidad o la imposibilidad de desarrollar y transmitir sus saberes ancestrales" (art. 8 del Decreto Ley 4635 de 2011). Además, la presentación del decreto menciona que los pueblos indígenas consideran el territorio como víctima, y enfatiza que este "hecho novedoso da cuenta del reconocimiento sociopolítico a la diversidad de epistemologías y de relaciones entre el hombre y la Madre tierra que prima con sus diferentes variantes en el pensamiento indígena" (Presentación del Decreto Ley 4633 de 2011). Si los daños culturales están integrados en el derecho positivo colombiano, algunas reivindicaciones de estos grupos fueron excluidas, como por ejemplo, justamente, el reconocimiento de la Madre Tierra como víctima9 .

Según estos criterios de justicia étnica colectiva, la reparación adecuada a favor de una familia indígena desplazada, por ejemplo, corresponde a la restitución de sus tierras con la posibilidad de gozar efectivamente de los derechos culturales correspondientes. En los casos en que la restitución sea imposible, y en la medida en que las compensaciones monetarias pueden ser prohibidas para respetar las normas de una comunidad ${ }^{10}$, otras formas de compensación deben necesariamente ser examinadas directamente con las comunidades. La concepción de las reparaciones a partir de las vivencias de

9 El territorio finalmente no ha sido incluido en la categoría de víctima por el artículo 3 del Decreto Ley 4633, "sin perjuicio al vínculo específico y colectivo que vincula las víctimas con la Madre-Tierra".

10 Los decretos leyes 4633, 4634 y 4635 prohíben las compensaciones monetarias de los "daños colectivos territoriales" por ser consideradas como nocivas para la armonía de la colectividad. 
los grupos de víctimas, y no a partir de criterios objetivos previstos de manera abstracta por un derecho que aparece ajeno, parece devolver su sentido a la fórmula "enfoque diferencial".

\subsection{La consulta previa como vector de etnorreparaciones y de garantías de no repetición}

Esta participación de las víctimas en la concepción de las reparaciones se materializó a través del mecanismo de la consulta previa, libre e informada -mecanismo por otra parte obligatorio para todas las leyes o medidas administrativas capaces de afectar directa o indirectamente a las comunidades autóctonas y sus territorios (art. 6 de la Convención 169 de la Organización Internacional del Trabajo - оाт, 1989). A parte de la paradoja residente en el hecho de que la Ley de Víctimas no había sido objeto de consulta, sus decretos ley de aplicación impusieron la consulta previa: "el plan tomará en cuenta la ley de origen, la ley natural, el derecho mayor, el derecho propio y las cosmovisiones de cada pueblo y comunidades que serán reparados y deberán ser consultados de manera previa con metodologías que se definirán con las autoridades y organizaciones de los pueblos indígenas respectivos" (Unidad para la Atención y Reparación Integral a las Víctimas, oIM, 2011).

En su confirmación de los conceptos de etnorreparaciones, el Acuerdo Final contiene ambiciosos aportes sobre la consulta previa, que corresponden a reivindicaciones de las etnias indígenas: el punto 6.2.3 reafirma especialmente su carácter "principal y no subsidiario", instaura el derecho a la "objeción cultural como garantía de no repetición" y, sobre todo, eleva el consentimiento libre, previo e informado al mismo nivel que la consulta previa en cuanto a su carácter obligatorio. Sin embargo, según la mayoría de las interpretaciones del Convenio 169 de la OIT, solo el mecanismo de la consulta presenta un alcance obligatorio, y no el resultado; se trata de un avance mayor del derecho de los pueblos autóctonos hacia la autodeterminación, concepto clave para la no repetición de los hechos que marcaron el conflicto.

En cuanto al primer punto de las negociaciones sobre el desarrollo agrario propio de las comunidades, el Capítulo étnico dota el "enfoque territorial" de una perspectiva étnica y cultural, ratificando la noción de "etnodesarrollo". Por otra parte, aumenta el margen de maniobra de los pueblos en conflictos vinculados con el territorio: "En la creación de mecanismos de resolución de conflictos de tenencia y uso y del fortalecimiento de la producción alimentaria participaran los pueblos y comunidades étnicas con sus organizaciones representativas cuando se trate de conflictos que comprometen sus derechos" (Acuerdo Final, p. 207). El Capítulo étnico se propone, también, reforzar la autonomía de los grupos minoritarios con el apoyo de sistemas de seguridad propios como la Guardia Indígena, encargada de proteger sus territorios. Además, prevé un desminado prioritario de estos últimos. 
E incluye asimismo garantías culturales innovadoras en un contexto mayoritariamente represivo respecto del problema de las drogas ilícitas. Prevé una acción que preserva las costumbres ancestrales del uso de plantas catalogadas como de uso ilícito. Los territorios de cultivos de coca deberían así ser tratados en el respeto de los pueblos y específicamente de los que están en vías de extinción. En fin, la misma firma del conjunto de los acuerdos entre las FARC-EP y el Gobierno favorece la restitución de tierras a familias desplazadas, que solo podrá materializarse una vez desmovilizados los grupos armados ilegales. Si el territorio no se pacifica efectivamente, estas medidas se quedarán meramente en aspiraciones condicionadas.

\section{CONCLUSIONES}

Todas estas medidas ilustran ciertas victorias para las minorías étnicas y culturales. Mas allá de las garantías que el texto prevé, esta transición conlleva la esperanza de una evolución en las relaciones de poder entre el derecho del Estado y las juridicidades alternativas. Las normas paralelas -de las FARC, de las minorías étnicas y de género-, una vez legitimadas por medio de los Acuerdos, son capaces de enriquecer la sociedad del posconflicto. El reto es entonces que los derechos propios, una vez afirmados, se vuelvan vectores de emancipación de los grupos étnicos y culturales.

Nos podemos preguntar no obstante si la promoción del derecho propio en los Acuerdos indica una confirmación del pluralismo jurídico en Colombia, o al contrario, una cooptación por un orden jurídico unitario de las normatividades alternativas. En este sentido, la proeza de los representantes indígenas con la inclusión del Capítulo étnico confiere un alcance ambivalente al Acuerdo. Nombrar a las víctimas en todas sus particularidades, y los perjuicios sufridos por el medio ambiente, por ejemplo, constituye un primer paso en contra de la invisibilización de sus derechos. Sin embargo, en la medida en que los pueblos indígenas hacen su lenguaje entendible para los operadores de la justicia estatal, y aquellos empiezan a hacer uso de este mismo lenguaje, el Estado adquiere control sobre los objetos designados por las cosmovisiones indígenas. En ese sentido, el politólogo Carlos Andrés Baquero llama la atención sobre los riesgos de la apropiación de los términos propios de las minorías por el Estado: "En ese proceso se logra más visibilización y más control pues, cuando se nombra, al mismo tiempo se libera y se domestica" (Baquero Díaz, 2015). Por otra parte, las poblaciones marginalizadas en busca de reconocimiento en el discurso mayoritario tuvieron que adaptar sus peticiones al lenguaje estatal, corriendo el riesgo de que sus reivindicaciones pierdan en esta traducción su esencia.

Así, si el Estado sigue siendo el garante último de este pluralismo jurídico y lo limita, ¿en que medida los derechos acordados a pueblos étnicos en los Acuerdos son vectores de emancipación de los pueblos? Más que el 
anuncio de un verdadero pluralismo jurídico, estos reconocimientos remiten a una rama del derecho estatal, el derecho de las minorías, que sigue siendo determinado de manera centralizada.

\section{REFERENCIAS}

ArizA, R. (2010). Peritaje socio-antropológico-jurídico y administración de la justicia intercultural en Colombia. Acceso a la justicia de los pueblos indígenas: los peritajes culturales y la visión de pobreza desde su cosmovisión. Instituto Interamericano de Derechos Humanos, 15.

Baquero Díaz, C. A. (2015). La magia de las palabras. Dejusticia, 15 de diciembre de 2015. Disponible en: http://www.dejusticia.org/\#!/actividad/2976 [Consulta: 15 de julio de 2016].

CONAFro. (2014). Declaración Política: Primer Encuentro de la Coordinación Nacional de Organizaciones y Comunidades Afrodescendientes, Medellín, 3 de diciembre.

Decretos Ley 4633, 4634 y 4635 del 9 de diciembre de 2011 en aplicación de la Ley 748 de 2011, "Ley de Víctimas y de Restitución de Tierras".

García Villegas, M.; De Sousa Santos, B. et al. (2001). El caleidoscopio de las justicias en Colombia. Bogotá: Siglo del Hombre Editores.

GutiéRrez Ramírez, L. M. (2015). Inventer la paix après un demi-siècle de conflit armé. Étude sur les discours de la justice transitionnelle en Colombie. Anayasa Hukuku Dergisi / Journal of Constitutional Law / Revue de Droit Constitutionnel. Vol. 4, n. ${ }^{\circ}$ 8, 63-84.

Gutiérrez Quevedo, M. (2013). Les mémoires occultées en Colombie. La Revue des droits de l'homme. 2, 2012. Disponible en: http://revdh.revues.org/260. [Consulta: 10 de agosto de 2016].

El Espectador. No hay puente entre la justicia transicional y la indígena. Feliciano Valencia. Disponible en: http://colombia2020.elespectador.com/justicia/no-hay-puente-entre-lajusticia-transicional-y-la-indigena-feliciano-valencia [Consulta: 8 de agosto de 2016].

Lefranc, S. (2013). Amérique latine et reste du monde: les voyages internationaux de la "justice transitionnelle". La Revue des droits de l'homme. 2, 2012, diciembre. Disponible en: http://revdh.revues.org/312 [Consulta: 30 de junio de 2016].

Mesas de Conversaciones. (2016). Comunicado conjunto n. ${ }^{\circ}$ 73, La Habana, 2 de junio de 2016. Disponible en: http://www.altocomisionadoparalapaz.gov.co/mesadeconversaciones/PDF/ comunicado-conjunto-73-1464879298.-la-habana-2-de-junio-de-2016-1464879298.pdf

Ministerio de Justicia y el Derecho, Escuela de Derecho Propio del Pueblo de los Pastos. (2015). Jurisdicción Especial Indígena y diálogo entre jurisdicciones. Instituto Interamericano de Derechos Humanos.

Ocqueteau, F.; Belley, J. G. et al. (1997). Le droit soluble, contributions québécoises à l'étude de l'internormativité. Genèses, 26. Représentations nationales et pouvoirs d'État, 165-166. 
Organizacion Internacional del Trabajo (oit). Convención 169 de la (oit) de 1989 sobre los pueblos indígenas y tribales. Disponible en: http://www.ilo.org/dyn/normlex/es/f? p=NORMLEXPUB:12100:0::NO::P12100_ILO_CODE:C169

Organización Nacional Indígena de Colombia (onic). (2016). Propuesta de Capítulo Étnico de la Comisión Étnica para la Paz y la Defensa de los Derechos Territoriales a la Mesa de Conversaciones de Paz de La Habana. Disponible en: http://www.onic. org.co/comunicados-onic/1386-propuesta-de-capitulo-etnico-de-la-comision-etnicapara-la-paz-y-la-defensa-de-los-derechos-territoriales-a-la-mesa-de-conversaciones. [Consulta: 19 de agosto de 2016].

Rodríguez Garavito, C. y Lam, Y. (2011). Etnorreparaciones: la justicia étnica colectiva y la reparación a pueblos indígenas y comunidades afrodescendientes en Colombia. Bogotá: Dejusticia, 2011.

Valencia Villa, H. (2012). Cartas de batalla. Una crítica del constitucionalismo colombiano. Bogotá: Panamericana.

Verdad Abierta. (2016). Acuerdo con las FARC tiene capítulo étnico. Disponible en: http:// www.verdadabierta.com/procesos-de-paz/farc/6377-indigenas-y-afros-a-la-expectativa. [Consulta: 26 de agosto de 2016].

Unidad para la Atención y Reparación Integral a las Víctimas, oim, Prosperidad para Todos. Gaviria Betancur, P. (dir.). (2011). Agenda Ruta de Reparación colectiva para grupos étnicos en el marco de los Decretos Ley 4633, 4634 y 4635 de 2011. 